Radial and Nonradial Pulsations as Probes of Stellar Physics

ASP Conference Series, Vol. 259, 2002

C. Aerts, T.R. Bedding, 83 J. Christensen-Dalsgaard, eds.

\title{
Non-Linear Radial Pulsation Models for the Early-Type Helium Stars V652 Her and BX Cir
}

\author{
P. Montañés Rodríguez, C. S. Jeffery \\ Armagh Observatory, Armagh, Northern Ireland, UK
}

\begin{abstract}
Hydrogen-deficient early-type stars have an extremely high surface helium abundance ( $>98 \%$ by number) and have represented a considerable challenge to stellar evolution theory. Recent work supports the view that they are the product of a merger between two white dwarfs.

All very luminous stars $(\log L / M>4)$ are believed to pulsate, but at effective temperatures around $20000 \mathrm{~K}$, helium stars with lower luminosities $(\log L / M<3)$ are also known to pulsate due to iron-group bump instability. The two known cases are V652 Her and BX Cir. Their observed periods are in good agreement with linear theory. Recently high-resolution spectroscopic observations were used to measure the stellar dimensions and radial velocity curves with high precision.

A hydrodynamic code including recent OPAL opacity data has been used to construct non-linear models of the pulsations of these two stars. The results impose additional constraints on those stellar dimensions, including mass, which remain poorly determined by observation.
\end{abstract}

\section{Introduction}

Extreme helium early-type stars are located between 10000 and $25000 \mathrm{~K}$ in the H-R diagram with $\log L / \mathrm{L}_{\odot} \gtrsim 3$. Although this locus coincides with that of massive main sequence stars, they are low mass stars $\left(\lesssim 1.0 \mathrm{M}_{\odot}\right)$ in an advanced stage of evolution. Several theories have been proposed to explain their evolutionary origin, including the merger of two helium white dwarfs (Saio \& Jeffery, 2000). These evolution models strongly depend on the stellar parameters, in particular, the stellar mass and metallicity have to be accurately known. Thus a precise measurement of the stellar dimensions is required.

At present just BX Cir and V652 Her have been identified as purely radial pulsators, excited by the $\kappa$-mechanism due to the iron-group opacity bump (Saio, 1995). Recent observations of V652 Her (Jeffery et al., 2001) have provided a lower value for its mass and a more accurate measurement of its abundances. New observations have also provided better estimates of the mass and effective temperature of BX Cir (Woolf \& Jeffery, 2000).

We attempt to reproduce accurately the observed radial velocity and luminosity curves and to constrain the stellar parameters by comparing these non-linear models with recent observational data. 
Table 1. Observational parameters for V652 Her and BX Cir.

\begin{tabular}{lll} 
& V652 Her ${ }^{1}$ & BX Cir \\
\hline$\Pi / \mathrm{d}$ & 0.108 & $0.1066^{2}$ \\
$M / \mathrm{M}_{\odot}$ & $0.59 \pm 0.1$ & $0.42 \pm 0.12^{3}$ \\
$T_{\text {eff }} / \mathrm{kK}$ & $20.95 \pm 0.07$ & $23.30 \pm 0.7^{4}$ \\
$R / \mathrm{R}_{\odot}$ & $2.31 \pm 0.02$ & $2.31 \pm 0.10^{3}$ \\
$\log L / \mathrm{L}_{\odot}$ & $2.96 \pm 1.15$ & $3.15 \pm 0.09^{-2}$ \\
$\log g / \mathrm{cm} \mathrm{s}^{-2}$ & $3.46 \pm 0.05$ & $3.35 \pm 0.1^{4}$ \\
$n_{\mathrm{H}}$ & 0.01159 & $0.00015^{4}$ \\
$n_{\mathrm{He}}$ & 0.98626 & $0.99590^{4}$ \\
$n_{\mathrm{C}}$ & $5.55 \times 10^{-5}$ & $0.00301^{4}$ \\
$n_{\mathrm{N}}$ & 0.00139 & $0.00052^{4}$ \\
$n_{\mathrm{Fe}}$ & $3.12 \times 10^{-5}$ & $9.51 \times 10^{-6} 4$ \\
$n_{\mathrm{Z}}$ & 0.00216 & $0.00394^{4}$ \\
\hline Jeffery et al. (2001) & \multicolumn{2}{c}{${ }^{2}$ Kilkenny et al. (1999) } \\
${ }^{3}$ Woolf \& Jeffery (2000) & ${ }^{4}$ Drilling et al. (1998)
\end{tabular}

\section{Non-linear pulsation code.}

A non-linear pulsation code (Bridger, 1984; Christy, 1967; Montañés Rodríguez \& Jeffery, 2001b) has been used to model radial oscillations. Hydrodynamic equations are solved explicitly for each temporal step and the thermodynamic equations are solved implicitly.

Initially a static model envelope is calculated (convection and energy generation are ignored and chemical composition is assumed to be constant). This static model is perturbed using a velocity profile. An artificial viscosity is introduced as a dissipative pressure, as in Stellingwerf (1975), with the viscosity parameters $\mathrm{C}_{\mathrm{Q}}=4.0$ and $\alpha_{\mathrm{v}}=0.1$.

The opacity was calculated using hydrogen-deficient (Alexander, 1994) and recent OPAL (Iglesias \& Rogers, 1996) opacity tables. These were initially generated in the OPAL Web site and have a solar mixture of metals with enhanced nitrogen or carbon.

\section{Models}

We investigated families of models obeying the equation $\log L=4 \log T_{\text {eff }}+$ $a_{1}$, where $a_{1}=\log \left(4 \pi \sigma(\Pi / Q)^{4 / 3} M^{2 / 3}\right)$. This fixed the periods of all models to $\Pi=0.107 \mathrm{~d} \pm 0.002$. Models were calculated with masses in the range 0.38 $1.16 \mathrm{M}_{\odot}$ and chemical mixtures shown in Table 2. Their positions in the H-R diagram are shown in Fig. 1.

\section{Conclusions}

Several models were found to reproduce with good accuracy the observed velocity and luminosity curves (see Fig. 2). For V652 Her stellar parameters of the 

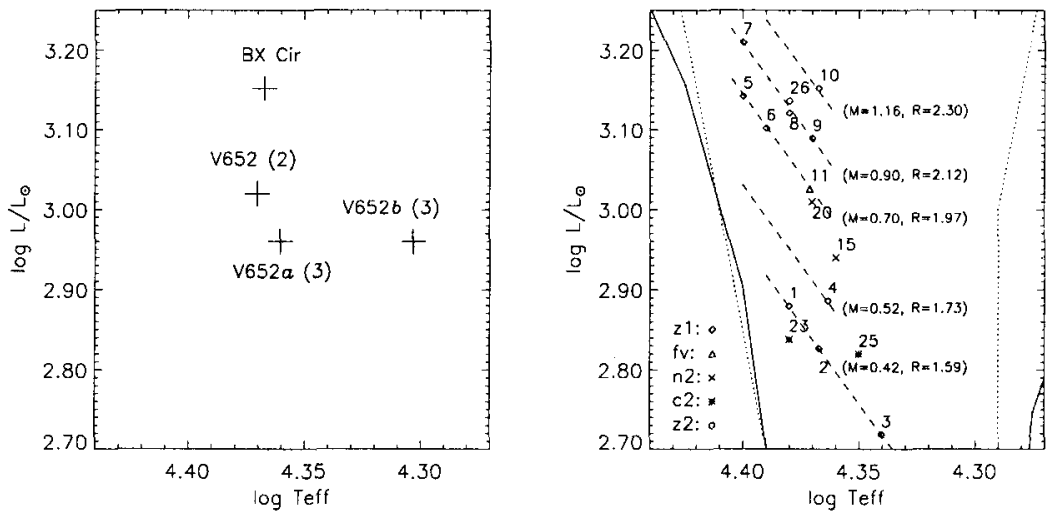

Figure 1. Location of V652 Her and BX Cir according to recent observations (left hand panel) and models calculated (right hand panel). Different symbols show the chemical mixtures used (Table 2). The iron-group bump instability strips for $0.5 \mathrm{M}_{\odot}$ (solid lines) and 0.7 $\mathrm{M}_{\odot}$ (dotted lines) for metallicity $\mathrm{Z}=0.01$ are also shown.
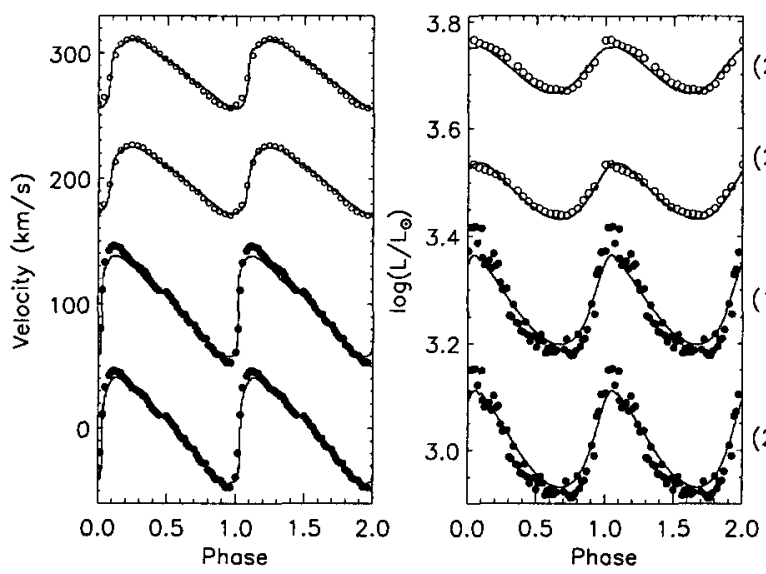

(23) $4.38 \quad 0.38$

$4.18 \quad 0.0066$

(25) $4.35 \quad 0.50$

$4.18 \quad 0.0066$

Figure 2. Observed luminosity and velocity curves (points) and the best fits models. Velocity curves were transformed into the stellar rest frame multiplying by -1.402 (projection factor for helium-rich early-type stars, Montañés Rodríguez \& Jeffery, 2001a). Next to the model number are written $\log T_{\text {eff }}$, stellar mass $\left(\mathrm{M}_{\odot}\right)$, iron abundance $\left(n_{\mathrm{Fe}} / 10^{-5}\right)$ and total abundance of metals $\left(n_{\mathrm{Z}}\right)$. 
Table 2. Adopted relative abundances by number.

$\begin{array}{cccccccc} & \text { fv } & \mathrm{n} 1 & \mathrm{n} 2 & \mathrm{c} 1 & \mathrm{c} 2 & \mathrm{z} 1 & \mathrm{z} 2 \\ n_{\mathrm{H}} & 0.0060 & 0.0115 & 0.0076 & 0.0000 & 0.0000 & 0.0000 & 0.0000 \\ n_{\mathrm{He}} & 0.9900 & 0.9862 & 0.9883 & 0.9961 & 0.9934 & 0.9976 & 0.9952 \\ n_{\mathrm{N}} & 0.0020 & 0.0015 & 0.0027 & 0.0003 & 0.0005 & 0.0002 & 0.0003 \\ n_{\mathrm{C}} & 0.0005 & 7.0 \times 10^{-5} & 0.0001 & 0.0029 & 0.0048 & 0.0006 & 0.0012 \\ n_{\mathrm{Fe}} / 10^{-5} & 4.72 & 2.37 & 4.17 & 2.49 & 4.18 & 5.19 & 10.45 \\ n_{\mathrm{Z}} & 0.0040 & 0.0023 & 0.0041 & 0.0039 & 0.0066 & 0.0024 & 0.0048\end{array}$

best fits were close to those previously provided by Fadeyev \& Lynas-Gray (1996) but with lower helium, carbon and iron abundances (model 20), closer to that given by recent observations. For $T_{\text {eff }} \sim 23400 \mathrm{~K}$, a mass of $0.70 \mathrm{M}_{\odot}$ is necessary to reproduce the observed velocity and luminosity curves. The attempt to reproduce the observed curves with a lower mass (around $0.59-0.67 \mathrm{M}_{\odot}$ ), closer to that recently observed, was not successful. However, final results are within the error limits of the measurements.

For BX Cir, observed velocity and luminosity curves have been well reproduced (model 25) with stellar masses within the error limits of the observations. A minimum mass equal to $0.38 \mathrm{M}_{\odot}$ for $23900 \mathrm{~K}$ (this value is within the observational error) and a maximum mass equal to $0.50 \mathrm{M}_{\odot}$ for $22300 \mathrm{~K}$ (smaller than the observed effective temperature) were found. Within these limits, an increase in stellar mass required a corresponding decrease in effective temperature to obtain the observed velocity and luminosity amplitudes.

\section{References}

Alexander, D.R. \& Ferguson, J.W. 1994, ApJ, 437, 879A

Bridger, A. 1984, in Cepheids: Theory \& Observations, (Knudsen), 246

Christy, F. 1966, ARA\&A, 4, 353

Drilling, J.S., Jeffery, C.S., \& Heber, U. 1998, A\&A, 329, 1019

Fadeyev, Y.A. \& Lynas-Gray, A.E. 1996, MNRAS, 280, 427

Iglesias, C.A. \& Rogers F.J. 1996, ApJ, 464, 943

Jeffery, C.S., Hill, P.W., \& Heber, U. 1999, A\&A, 346, 491

Jeffery, C.S., Woolf, V.M., \& Pollacco, D. 2001, A\&A, in press

Kilkenny, D., Koen, C., Jeffery, C.S., Hill, N.C., \& O'Donoghue, D. 1999, MNRAS, 310,1999

Montañés Rodríguez, P. \& Jeffery, C.S. 2001, A\&A, 375, 411

Montañés Rodríguez, P. \& Jeffery, C.S. 2001, A\&A, submitted

Saio, H. 1995, MNRAS, 277, 1393

Saio, H. \& Jeffery, C.S. 2000, MNRAS, 313, 671

Stellingwerf, R.F. 1975, ApJ, 195, 441

Woolf, V.M. \& Jeffery, C.S. 2000, A\&A, 358, 1001 